\title{
Similar effects of medial supramammillary or systemic injection of chlordiazepoxide on both theta frequency and fixed-interval responding
}

\author{
MARY-ANNE WOODNORTH and NEIL MCNAUGHTON \\ University of Otago, Dunedin, New Zealand
}

\begin{abstract}
The frequency of theta activity may be important for hippocampal function. Anxiolytic drugs reduce theta frequency and have behavioral effects that are similar to those of hippocampal lesions. The effect of the anxiolytic benzodiazepine chlordiazepoxide (CDP) on theta frequency is partially mediated by the medial supramammillary nucleus (mSuM), part of an ascending theta-activatingsystem. Rats were trained on the hippocampal-sensitive fixed-interval 60-sec schedule (FI60). CDP (5 mg/kg i.p.) released responding suppressed by nonreward, seen as increased leverpressing, and reduced theta frequency concurrently. Microinfusion of CDP ( $20 \mu \mathrm{g}$ in $0.5 \mu \mathrm{l}$ saline) into mSuM had as large effects on both frequency and behavior. Other nuclei mediate the benzodiazepine reduction of theta frequency in the open field and the water maze. But the mSuM appears to be the major, if not sole, nucleus controlling theta frequency and, so, hippocampal-mediated behavioral inhibition in the FI60 lever task.
\end{abstract}

Rhythmical slow activity, or theta rhythm, is a highvoltage extracellular waveform present in the hippocampal formation of a number of mammalian species (Black \& Young, 1972; Kemp \& Kaada, 1975; Sainsbury, 1970; Stewart \& Fox, 1989b; Winson, 1972), including humans (Sano, Mayanagi, Sekino, Ogashiwa, \& Ishijima, 1970). In the rat, theta is prominent during voluntary movements (Slawinska \& Kasicki, 1998; Vanderwolf, 1969) and has been associated with basic psychological functions, such as arousal (Green \& Arduini, 1954), orienting (Grastyan, Lissak, Madarasz, \& Donhoffer, 1959), attention (Bennett, 1975), sensorimotor processing (Bland, 1986), and sensory inhibition (Sainsbury, 1998). Numerous studies have also linked theta with higher order cognitive processes (AmmassariTeule, Maho, \& Sara, 1991; Destrade, 1982; Elazar \& Adey, 1967; Givens \& Olton, 1994; Grastyan, Karmos, Vereczkey, \& Kellenyi, 1966; Landfield, 1977; Landfield, McGaugh, \& Tusa, 1972; Wetzel, Ott, \& Matthies, 1977; Winson, 1978), and with emotion (Graeff, Quintero, \& Gray, 1980; Gray, 1972; Montoya, Heynan, Faris, \& Sainsbury, 1989; Snape, Grigoryan, Sinden, \& Gray, 1996; Williams \& Gray, 1996). What is often common to these latter two perspectives is the idea that the specific frequency of theta is critical for the behavioral output. Several major theories identify the frequency at which theta occurs as important for the overall functioning of the hippocampus (Bland, 1986; Miller, 1991). In particular, it has been proposed that the capacity of anxiolytic drugs to alter the control of theta frequency (Gray \& Ball, 1970; McNaughton, Richardson, \&

Correspondence concerning this article should be addressed to N. McNaughton, Department of Psychology, University of Otago, P. O. Box 56, Dunedin, New Zealand (e-mail: nmcn@ psy.otago.ac.nz).
Gore, 1986; McNaughton \& Sedgwick, 1978; Zhu \& McNaughton, 1994) implicates the hippocampus in the behavioral effects of these drugs and, hence, in the control of anxiety (Gray \& McNaughton, 2000).

High-frequency stimulation of brainstem sites elicits theta (Green \& Arduini, 1954; Petsche, Stumpf, \& Gogolak, 1962), the most effective site being the nucleus reticularis pontis oralis (RPO; Vertes, 1980). Theta is paced by cells in the medial septum/vertical limb of the diagonal band of Broca (MS/vDBB). A population of MS/vDBB neurons fire in a constant phase relationship with theta (Apostol \& Creutzfeldt, 1974; Brazhnik \& Vinogradova, 1986; Gogolak, Stumpf, Petsche, \& Sterc, 1968; Petsche et al., 1962; Stewart \& Fox, 1989a), and lesions of the MS/vDBB or its main afferent pathway, the fimbria/fornix, abolish theta (Buzsaki, Leung, \& Vanderwolf, 1983; Green \& Arduini, 1954; Rawlins, Feldon, \& Gray, 1979; Sainsbury \& Bland, 1981; Stumpf, 1965). It was once thought that the MS/ vDBB also encoded the frequency of theta. However, it has been demonstrated that the septal region receives only sparse input from the RPO (Vertes, 1986), and anatomical and physiological evidence suggests that information received by the MS/vDBB has already been coded for frequency elsewhere.

Kirk and McNaughton $(1991,1993)$ proposed that the medial supramammillary nucleus ( $\mathrm{mSuM})$ is one site where tonic (intensity) information from the brainstem is transduced to phasic (frequency) information. They found that, during RPO stimulation, cells in the mSuM of anesthetized rats fire rhythmically and in phase with ongoing theta and persist in firing rhythmically after the MS/vDBB is inactivated (Kirk \& McNaughton, 1991). With procaine mapping and RPO stimulation (Kirk \& McNaughton, 1993), theta amplitude, but not frequency, was reduced by procaine 
rostral to the mSuM, whereas frequency was reduced by procaine in the pathways from the RPO to the mSuM. Procaine in the $\mathrm{mSuM}$ itself reduced both the frequency and amplitude of theta. A transduction role for the mSuM of brainstem impulses bound for the medial septum is consistent with tracing studies that have identified projections to the mSuM from the RPO (Vertes \& Martin, 1988) and other brainstem structures (Hayakawa, Ito, \& Zyo, 1993) and from the $\mathrm{mSuM}$ to the medial septum via the median forebrain bundle (Borhegyi, Magloczky, Acsady, \& Freund, 1998; Shepard, Mihailoff, \& German, 1988; Vertes, 1988, 1992).

In awake rats, the anxiolytic benzodiazepine chlordiazepoxide(CDP) injected into the $\mathrm{mSuM}$ reduced the frequency of theta elicited by RPO stimulation, but to a lesser degree than it did under anesthesia (McNaughton et al., 1995). These findings have two important implications. First, the neural mechanism of theta frequency control by the mSuM is likely to be GABAergic inhibition, which benzodiazepinesenhance (Olsen, 1982). Second, frequency is not determined solely by the $\mathrm{mSuM}$ in the awake rat, whereas it appears to be in the anesthetized preparation. Another nucleus or other nuclei must augment frequency control by the mSuM under normal behavioral conditions, and recent results from our laboratory suggest that the specific sites involved may vary according to conditions (Woodnorth, Kyd, Logan, Long, \& McNaughton, 2002). Nonetheless, according to the view that theta frequency is important for hippocampal functioning, the mSuM would be expected to exert some influence over behaviors under hippocampal control.

To date, only one mSuM study has directly compared performance on a hippocampal-sensitive task with ongoing theta. Pan and McNaughton (1997) found that in the Morris water maze, intracranial injection of CDP into the $\mathrm{mSuM}$ produced only a small reduction in the frequency of theta and a similarly modest impairment of spatial learning. They argued that the broad degree to which theta is affected will be associated with the level of behavioral dysfunction in tasks requiring the hippocampus, since in the same and previous experiments (McNaughton et al., 1995; McNaughton \& Morris, 1987), the size of treatment effects on theta frequency matched the size of their effects on spatial learning.

The aim of the present experiment was to investigate the effects produced on behavior and theta frequency by injecting CDP into the mSuM in a task involving nonspatial behavioral inhibition(Gray \& McNaughton, 2000), as opposed to a task requiring spatial memory or exploratory movement. One such task is acquisition of a fixed-interval (FI) schedule, where animals typically learn to suppress their responding during periods of frustration generated by nonreward. FI responding is released from suppression after systemic injection of both classical anxiolytic drugs such as CDP and novel anxiolytic drugs such as buspirone (Panickar \& McNaughton, 1991; Zhu \& McNaughton, 1995). Both classes of anxiolytics reliably reduce theta frequency (McNaughton \& Coop, 1991; McNaughton et al.,
1986). FI responding is also released in rats with septal or hippocampal lesions (see Gray \& McNaughton, 1983). Since the $\mathrm{mSuM}$ is involved in emotion (Beck \& Fibiger, 1995; Sandner et al., 1993) as well as in the control of theta frequency, it was our prediction that CDP would act specifically at the $\mathrm{mSuM}$ during FI and that a systemic injection of the drug would thus produce similar effects on theta frequency and behavior.

\section{GENERAL METHOD}

\section{Animals}

Forty-one adult (250-550 g) male Sprague-Dawley rats were used for this study. They were obtained from the University of Otago Department of Laboratory Animal Sciences. The rats were housed individually in hanging wire-mesh cages. Ten days post surgery, the animals were gradually introduced to a 23 -h food deprivation schedule over 4 days. Subsequent to this, they were maintained on 23-h food deprivation for at least 10 days before the commencement of training. During training and testing, the animals were fed in their home cages for $1 \mathrm{~h}$ immediately after their daily session.

\section{Surgery}

All the rats were implanted with recording and stimulating electrodes. Those rats that were originally assigned to receive intracranial injections $(n=30)$ were also implanted with cannulae. Atropine sulphate $(25 \mathrm{mg} / \mathrm{kg})$ was administered prior to surgery in order to reduce respiratory congestion. Electrodes and cannulae were stereotaxically implanted under anesthesia with sodium pentobarbital $(60 \mathrm{mg} / \mathrm{kg})$. Recording electrodes consisted of two strands of Teflon-coated stainless steel wire (70- $\mu \mathrm{m}$ outside diameter), which were twisted together, with the tips separated by $2.0 \mathrm{~mm}$, and implanted in the subicular region of the dorsal hippocampus (bregma A-P 6.0, M-L 2.0, D-V 5.0). A stimulating electrode with a tip separation of $0.5 \mathrm{~mm}$ was implanted into the region of the nucleus pontis oralis (RPO) of the rostral pons (bregma A-P 7.0, M-L 1.6, D-V, 8.5). For those animals used for intracranial testing, a 25-gauge guide cannula was lowered either 8.0 or $8.5 \mathrm{~mm}$ toward the $\mathrm{mSuM}$, using coordinates $4.5 \mathrm{~mm}$ posterior to bregma and $0.9 \mathrm{~mm}$ lateral to the midline at an angle from the vertical of $6^{\circ}$. An obdurator (modified insect pin) was inserted into the guide cannula to prevent occlusion. All coordinates were determined with reference to the skull surface and were obtained from the stereotaxic atlas of Paxinos and Watson (1998). A ground electrode consisting of a length of uninsulated silver wire (0.25-mm diameter) was wound around a stainless steel skull screw. All the electrodes were inserted via Amphenol gold pins into a McIntyre miniconnector that was secured to the skull with dental cement.

\section{Drug and Injection Procedures}

Systemic injections. Rats receiving intraperitoneal (i.p.) injections were injected $10 \mathrm{~min}$ prior to testing. Injections were either saline $(0.9 \% \mathrm{NaCl} ; 1 \mathrm{ml} / \mathrm{kg})$ or CDP hydrochloride $(5 \mathrm{mg} / \mathrm{kg}$, dissolved in saline, $5 \mathrm{mg} / \mathrm{ml}$; Roche Laboratories).

Intracranial injections. Ten minutes prior to testing, the obdurator was removed from the guide cannula, and the injection cannula was inserted. The injection cannula consisted of silica capillary tubing (VS-140-40, Scientific Glass Engineering, U.K.; $140 \mu \mathrm{m}$ external diameter and $40 \mu \mathrm{m}$ internal diameter) with one end glued into a stainless steel collar. When the collar was flush against the guide cannula, the injection cannula extended $1 \mathrm{~mm}$ into the brain beyond the tip of the guide. Polythene tubing connected the injection cannula to a 10- $\mu 1$ Hamilton syringe, which was driven by an electrical microdrive. Drugs were infused at a volume of $0.5 \mu \mathrm{l}$ over a period of $2.5 \mathrm{~min}$, after which the injection cannula was removed and the obdurator was reinserted. The rats received either saline or CDP $(40 \mathrm{mg} /$ 
$\mathrm{ml}$, dissolved in saline). The movement of, and the lack of compression of, an air bubble in the polythene tubing was used to monitor the success of the infusions.

Owing to the brittle nature of the silica tubing and the difficulty of inserting this into the very small aperture of the guide cannula in a fully conscious rat, the silica injection cannulae occasionally snapped off in the guide, rendering further intracranial injections impossible. When this occurred before a rat had received a drug on the 1st day of FI training $(n=3)$, the animal was immediately reassigned to one of the systemic groups. When a cannula breakage occurred after Day 1 of FI training, the rat was retired from the study.

\section{Apparatus and Procedure}

Training and testing took place in a Campden Instruments Operant Chamber $(24.5 \times 22.5 \times 23 \mathrm{~cm})$, modified so that $45-\mathrm{mg}$ food pellets (Campden Instruments, U.K.) were delivered to an aluminum dish secured to the grid floor and a recording cable could extend through the ceiling into the chamber. The chamber was controlled by a BBC$\mathrm{B}$ microcomputer programmed in BBC BASIC with SPIDER realtime commands (Paul Fray, Cambridge, U.K.). Individual rats had their operant sessions at the same time on each day of the procedure.

Pretraining. Following 10 or more days of 23 -h food deprivation, the rats were trained on a noncontingent random time 30 -sec schedule, wherein single food pellets were delivered at intervals of between 1 and $60 \mathrm{sec}$ after the previous delivery. The rats received four 30-min pretraining sessions on consecutive days.

Continuous reinforcement. During 30-min continuous reinforcement (CRF) sessions, a lever was extended into the chamber, and each leverpress made by the rat was rewarded with a food pellet. On the 4th day of CRF and on every subsequent day of the procedure, the cable was suspended into the chamber and connected with the rats' head connectors. The rats received four CRF sessions on consecutive days unless, on the 4th day, their leverpress responding had dropped by more than $30 \%$ from the previous session. In these cases, a fifth CRF session was run the following day.

Fixed interval. For the remainder of the experiment the rats were trained on an FI 60-sec (FI60) schedule. In this schedule, the first lev- erpress response of the session is rewarded, and this response starts the first 60 -sec interval. The rats received a reward only after $60 \mathrm{sec}$ had elapsed since the last rewarded response, and each rewarded response reset the interval. Each FI session was 30 min long, and training continued for 8 days or until cannula breakage prevented further drug infusions.

\section{Theta Recording and Data Collection}

During the last CRF session and throughout FI training, EEG data were recorded from the hippocampus. Electrodes were connected via the McIntyre connector to a cable and a dual field effect transistor, preamplifier (Grass P511K, 1-30 Hz bandpass filter), and extracellular field activity was digitized at $100 \mathrm{~Hz}$ for subsequent analysis by an Acorn A5000 computer. The leverpress data were collected and analyzed through a parallel EEG channel. Both the EEG and the behavioral data were displayed on the Acorn computer during FI sessions, the first as a continuous waveform and the second as a series of discrete bars with rewarded responses represented at twice the height of nonrewarded responses.

\section{Histology}

The 27 rats that had received intracranial injections were deeply anesthetized (sodium pentobarbital) and were perfused transcardially with saline and then with $10 \%$ formalin. The brains were removed and kept in 30\% sucrose-formalin for 7 days. Frozen coronal sections $(60 \mu \mathrm{m})$ were mounted and stained with thionin. The positions of the cannula tips were reconstructed according to the atlas of Paxinos and Watson (1998). The animals were classif ied on the basis of the distance of tip location from the mSuM (see Figure 1). The radius of diffusion of drugs injected in a volume of $0.5 \mu 1$ is approximately $500 \mu \mathrm{m}$ (Myers, 1966), and this also appears to be the extent of the functional diffusion of drugs in the region of the $\mathrm{mSuM}$ (McNaughton et al., 1995). The animals were classified as $m S u M$ if the tip was in or within $500 \mu \mathrm{m}$ of the $\mathrm{mSuM}(4.16-4.80 \mathrm{~mm}$ posterior to bregma) or outside the $m S u M$ when the tip was remote from the boundary of the mSuM by $>500 \mu \mathrm{m}$ (see McNaughton et al., 1995). These outside the mSuM animals were included in the analyses

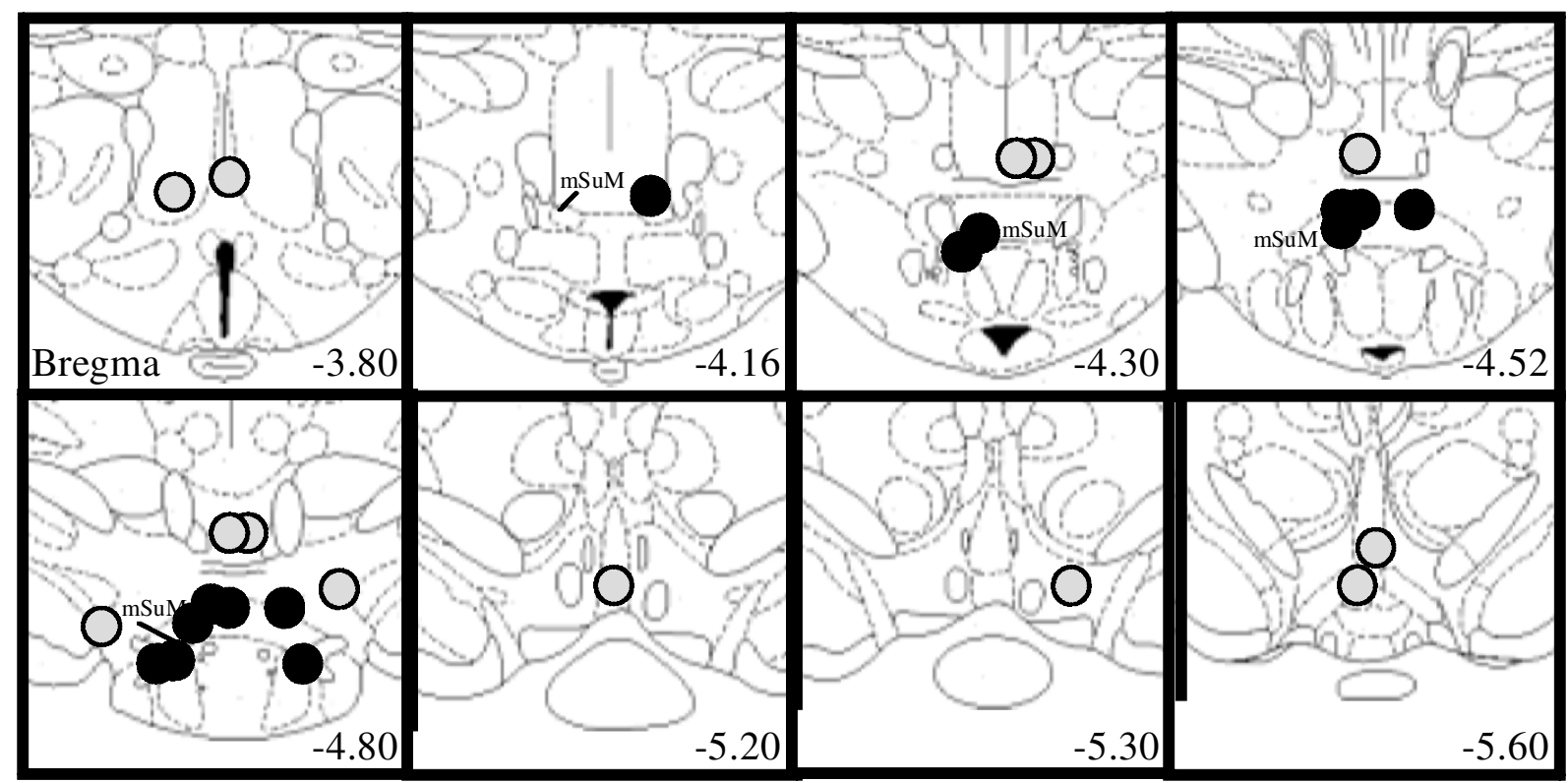

Figure 1. Cannula tip locations for 27 rats that had received one or more intracranial injections of CDP (20 $\mu \mathrm{g}$ in $0.5 \mu \mathrm{l}$ vehicle) or saline. Stained coronal sections $(60 \mu)$ were compared with the stereotaxic atlas of Paxinos and Watson (1998). Dark circles, placements in or $<500 \mu$ from the $\mathbf{m S u M}$; light circles, placements $>500 \mu$ outside of the $\mathbf{m S u M}$. 
Table 1

Numbers of Rats in Each Condition for the Behavioral

and Theta Frequency Analyses

\begin{tabular}{lcccccc}
\hline & \multicolumn{2}{c}{ FI Behavior } & & \multicolumn{2}{c}{ FI Theta Frequency } \\
\cline { 2 - 3 } \multicolumn{1}{c}{ Condition } & CDP & Saline & & CDP & Saline \\
\hline mSuM & 7 & 7 & & 4 & 5 \\
Outside the mSuM & 7 & 6 & & 7 & 3 \\
Systematic (i.p.) & 7 & 7 & & 5 & 5 \\
\hline
\end{tabular}

as anatomical controls for the effects of the drugs in the mSuM (see Table 1).

\section{Data Collection and Analysis}

Behavioral data. During FI sessions, the leverpresses were allocated to twelve 5-sec bins according to when they occurred in the interval. The total number of responses in each of the 12 bins was recorded for each rat for each day of FI. These data were transformed $\left(X^{\prime}=\operatorname{SQRT}[X+0.5]\right)$ to normalize the data distribution (Zar, 1974) and were submitted to a repeated measures analysis of variance. The drug received (CDP or saline) and the site of injection (mSuM, outside mSuM, or i.p.) for each animal were extracted as between-subjects factors. Days and interactions with days were extracted as between-days components, and the variation in response rates across bins and all interactions with bins were extracted as between-bins components. Polynomial contrasts were extracted for the site (linear and quadratic) and the days and bins (linear, quadratic, and cubic) factors (Snedecor \& Cochrane, 1967). Given the specific ordering chosen of levels of the factor of site of injection (mSuM, outside mSuM, i.p.), the quadratic component estimates variance common to $\mathrm{mSuM}$ and i.p. and not shared with outside $\mathrm{mSuM}$, whereas the linear component results from differences between $\mathrm{mSuM}$ and i.p., ignoring outside $\mathrm{mSuM}$. "Linear" and "quadratic," therefore, identify the method of extraction of the contrasts but are not the linearity or quadraticity of any underlying unidimensional factor.

EEG data. Only data from rats that produced a reliable EEG were analyzed (29 rats). A computer program selected the first leverpress made in each 10-sec bin of each 60-sec interval of the FI and analyzed $0.75 \mathrm{sec}$ of theta beginning $1 \mathrm{sec}$ before the leverpress. Frequency of theta was determined by an algorithm that detected peaks of successive theta waves and calculated frequency from the number of waves and the interval between the first and the last peaks. The criteria for theta were that the waveform should be sinusoidal, with a frequency of 4-12 Hz. Each sample was visually inspected during analysis. Waveforms that did not match these criteria were excluded. Correct peak detection was also confirmed visually for all the samples. Mean frequency for each of six 10-sec bins for $30 \mathrm{~min}$ of FI was calculated for each rat and was analyzed as above, with change in theta frequency across bins and interactions with bins analyzed as between-bins components.

\section{RESULTS}

\section{Fixed Interval Barpressing}

The effects of CDP at the three injection sites on FI responding are shown in Figure 2. It can be seen that the drug received and the site of injection both affected responding. The three saline groups produced similar rates of responding on individual days (Figure $2 \mathrm{~A}$ and $2 \mathrm{~B}$ ) and overall (Figure 2C). Responding by the rats that had CDP outside of the mSuM resembled that of the saline groups on Day 1, but, overall, appeared slightly below that of controls. CDP i.p. and CDP in the $\mathrm{mSuM}$ produced rates of re- sponding similar to one another and greater than those produced by saline and CDP outside the mSuM. The magnitude of these effects increased over the 4 days [drug $\times$ site: $\times$ days: $\operatorname{dev} \times$ quad $\times$ lin, $F(1,25)=9.24, p<.05$ ]. $\mathrm{A}$ post hoc analysis restricted to the i.p. and $\mathrm{mSuM}$ sites of injection revealed that there was no difference in overall responding between CDP i.p. and CDP in the $\mathrm{mSuM}$ [site: dev, $F(1,15)=0.427$, n.s.]. A post hoc analysis also yielded no difference in overall responding between the CDP and the saline animals with placements outside the mSuM [drug: $\operatorname{dev} \times \operatorname{lin}, F(1,15)=0.673$, n.s.]. The release of responding seen with CDP i.p. and $\mathrm{mSuM} / \mathrm{CDP}$ did not involve any change in the shape of the FI curve. Therefore, drug and site did not interact to produce any effect on bins.

\section{Theta Frequency}

Only data from the first 3 days of FI training could be analyzed, owing to missing values. Figure 3 shows the effects of CDP at the three sites of injection on the frequency of theta recorded $1 \mathrm{sec}$ prior to barpressing. Those rats that received saline in any site or CDP outside of the $\mathrm{mSuM}$ produced an average frequency of theta of between 6.8 and $7 \mathrm{~Hz}$. CDP i.p. and $\mathrm{mSuM} / \mathrm{CDP}$ reduced these frequencies to below 6.2 and $6.4 \mathrm{~Hz}$, respectively [drug $\times$ site: dev $\times$ quad, $F(1,8)=11.14, p<.05]$. No significant difference in overall theta frequency was found in post hoc analysis of the CDP i.p. and $\mathrm{mSuM} / \mathrm{CDP}$ groups [site: dev, $F(1,3)=0.0128$, n.s.]. Frequency was stable for all groups across days and bins.

\section{DISCUSSION}

In this experiment, the effects of a systemic injection of the benzodiazepine CDP on both behavior and theta frequency were reproduced in their entirety when the drug was injected into the $\mathrm{mSuM}$. As with previous experiments from this laboratory (Panickar \& McNaughton, 1991; Zhu \& McNaughton, 1995), CDP i.p. increased the rate of FI barpressing, relative to saline. Concurrent with the effect produced on responding, CDP reduced the frequency of the hippocampal theta rhythm. Although this effect of benzodiazepines on frequency in awake animals is well documentedin electrophysiologicaltests (McNaughton \& Coop, 1991; McNaughton et al., 1995; McNaughton et al., 1986), this is the first time, to our knowledge, that it has been demonstrated during an operant task used to model anxiety. When CDP was injected into or near the 

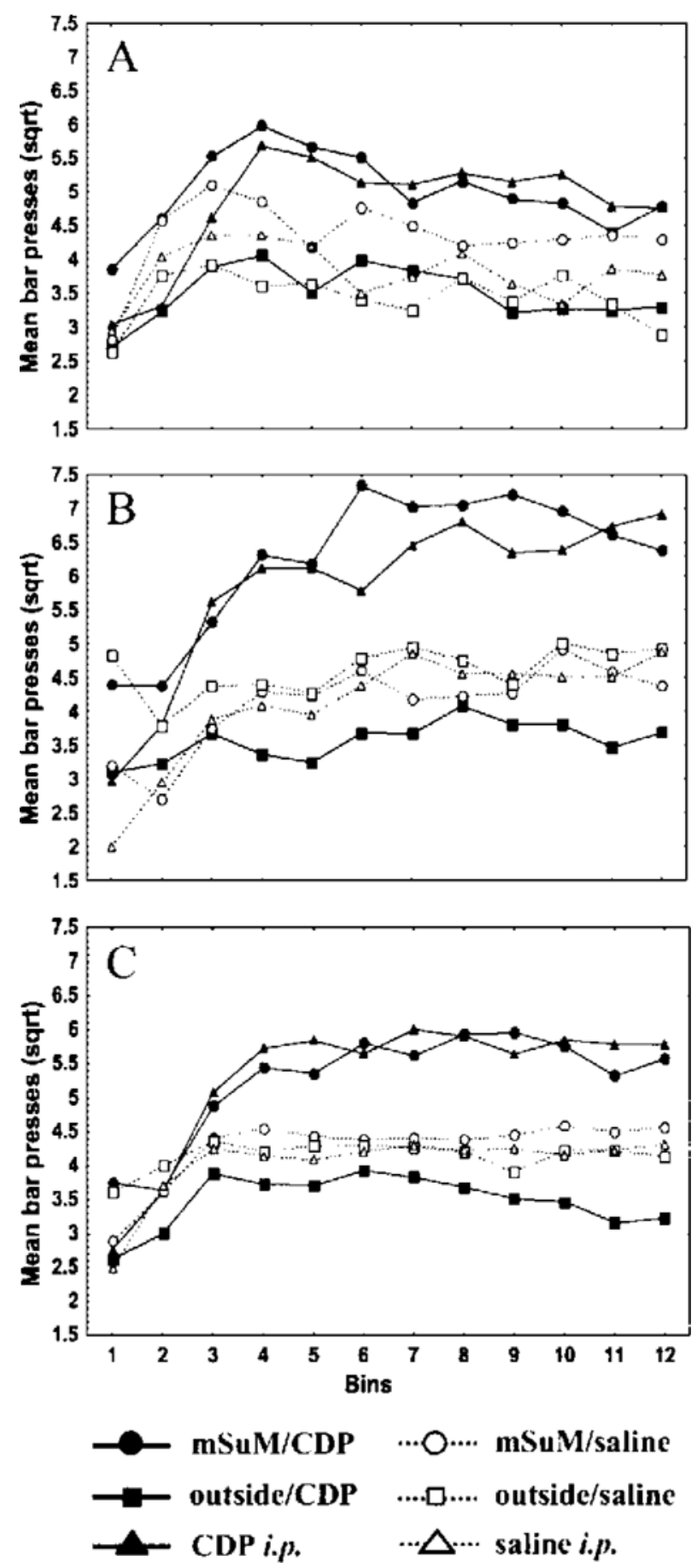

Figure 2. The effects of CDP (in $\mathrm{mSuM}$, outside $\mathrm{mSuM}$, or i.p.) on responding during acquisition of FI60 for (A) Day 1 and (B) Day 4 of training and for $(C)$ the average of Days 1-4. Data are SQRT $[X=0.5]$ of the mean number of responses binned according to latency since the last rewarded response. Note that the acceleration over the latter bins typically seen with this schedule ("scalloping") is missing on individual days and overall. It is possible that attachment with the recording cable may have interacted in some way with the schedule's control of the rats' behavior. However, these patterns of responding were identical in rats treated with i.p. saline or CDP and in rats treated with intracranial saline or CDP.
$\mathrm{mSuM}$, the effects on responding and on theta frequency were striking in their similarity to those produced by the i.p. injection. The magnitude of the release of FI responding by both groups, relative to controls, increased incrementally over days to a virtually identical degree. Theta frequency was analyzed across fewer days of training than was behavior, but on all of these days, both i.p. CDP and mSuM/ CDP produced a stable reduction of frequency. These findings suggest that the $\mathrm{mSuM}$ was mediating the effects of $\mathrm{CDP}$ on both theta frequency and barpressing during the FI schedule.

When CDP was injected into brain structures remote from the mSuM by more than $500 \mu \mathrm{m}$, neither of the effects of CDP i.p. were reproduced. Theta frequencies from animals that had received this treatment were indistinguishable from the saline controls. After 4 days of training, the FI responding of these animals may have been slightly suppressed, as compared with the saline groups, but was certainly not increased.

Consistent with McNaughton et al. (1995), we have found that the mSuM has an important role in determining theta frequency in the awake rat. According to the view that theta frequency is important for normal hippocampal functioning (Kirk, 1998; Gray \& McNaughton, 2000; Miller, 1991; Vinogradova, 1995), then, the mSuM is likely to interact with some functions of the hippocampus. Initial proof of this was obtained by Pan and McNaughton (1997), who found that benzodiazepine enhancement of GABAergic inhibition in the mSuM reduced theta frequency and impaired spatial learning in the Morris water maze. However, the size of both effects was much smaller than the size of systemically injected CDP. In the present experiment, the large reduction of theta frequency with $\mathrm{mSuM} / \mathrm{CDP}$ was associated with a specific change in the rate of FI barpressing, which is also controlled by the septohippocampal system (Beatty \& Schwartzbaum, 1968; Ellen \& Powell, 1962; Haddad \& Rabe, 1969; Manning \& McDonough, 1974). Together, these findings suggest that the hippocampus is capable of making functionally relevant distinctions between different frequencies of theta.

However, it is important to rule out the possibility that changes in the theta recorded during FI training were merely dependent on movement, which the hippocampus does not control. Hippocampal theta has a robust association with voluntary movements (Slawinska \& Kasicki, 1998; Kramis, Vanderwolf, \& Bland, 1975; Vanderwolf, 1969; Whishaw \& Vanderwolf, 1973), including leverpressing (Black \& Young, 1972). A number of observations support a relationship between movement or movement initiation and theta frequency (Bland \& Vanderwolf, 1972; Morris \& Hagan, 1983; Oddie, Stefanek, Kirk, \& Bland, 1996; Rivas, Gaztelu, \& Garcia-Austt, 1996; Slawinska \& Kasicki, 1998; Vanderwolf, 1969; Whishaw, Bland, \& Vanderwolf, 1972). In general, higher frequencies are associated with greater initial speed of movements and the preparation for such movements. Bland's (1986) sensorimotor model regards movement-related theta as being due to feedback to the hippocampus from systems involved in executing movement. 

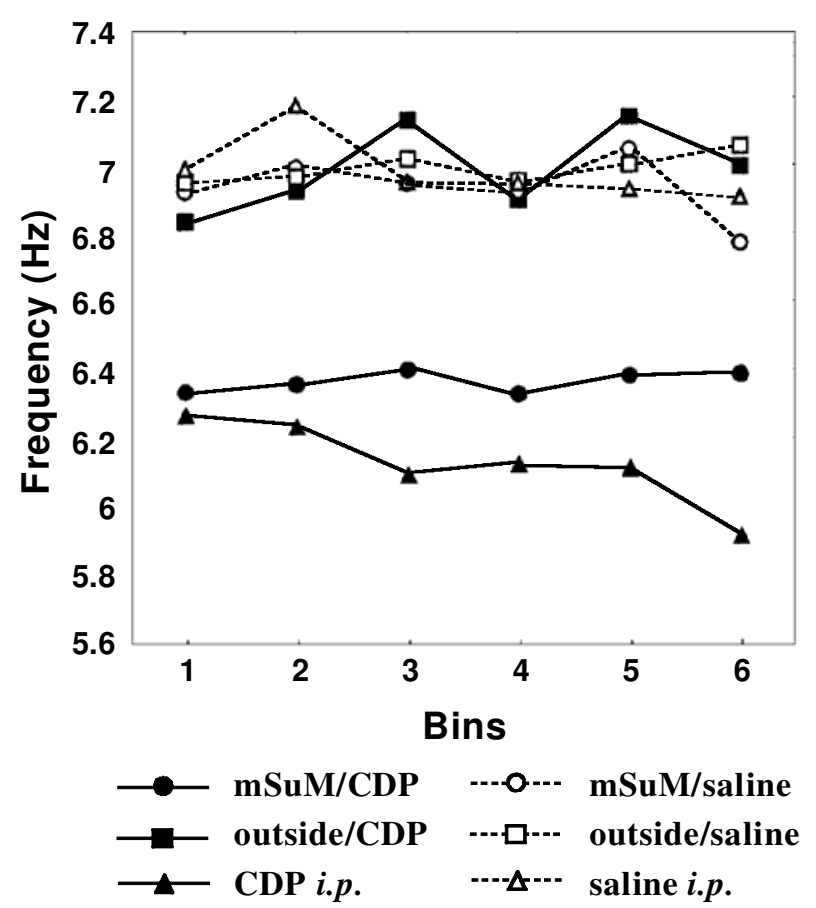

Figure 3. Effects of CDP (in the mSuM, outside the mSuM, or i.p.) on theta frequency during FI60. Data are the mean frequency (in hertz) of theta waves recorded in the second prior to the first barpress in each of six 10 -sec bins. Data are averaged over the first 3 days of FI60 training.

This elicited theta, in turn, provides movement systems with an update of their performance. In this experiment, theta was sampled prior to a stereotyped behavior (leverpressing). Because the force required for each press of the lever was equivalent and the range of behaviors possible immediately prior to responding limited, the behavioral response was effectively "clamped" (see Lee, Chrobak, Sik, Wiley, \& Buzsaki, 1994) during measurement of theta frequency. Therefore, group differences in theta frequency cannot easily be attributed to any motor hypothesis. Moreover, CDP i.p. and CDP in the mSuM increased the overall rate of leverpressing while reducing the frequency of theta associated with it. Theta frequency also, unlike movement, did not change noticeably during training.

The present results are more easily explained in terms of behavioral inhibition, rather than free movement. Behavioral inhibition is characterized in many operant tasks by the passive avoidance (suppression) of a prepotent response in the presence of punishment or nonreward (Gray \& McNaughton, 2000). Anxiolytic drugs that reduce theta frequency reduce behavioral inhibition in many tasks. Opposite effects on behavioral inhibition have also been obtained with different frequencies of theta produced by septal driving stimulation delivered prior to testing (Gray, 1972; Snape et al., 1996; Williams \& Gray, 1996). In our FI60 paradigm, barpressing was released by CDP during non- reward, and individual responses were associated with lower frequencies of concurrently recorded theta than were produced by controls. These findings are consistent with a role for the hippocampus, via theta, in the suppression of behavior during frustration or nonreward and, hence, in the control of anxiety (Gray \& McNaughton, 2000). In the case of anxiety as modeled by FI60, our results suggest that the benzodiazepine-sensitive component of theta frequency is determined largely, and perhaps exclusively, by the $\mathrm{mSuM}$. The mSuM is likely, therefore, to be one important site for the clinical effects of anti-anxiety drugs. However, sites that are sensitive to anxiolytic drugs in animal models are widely distributed in the brain, and in addition to the $\mathrm{mSuM}$, anxiolytic effects of intracranially administered benzodiazepines have been observed in dorsal and median raphe, dorsal periaqueductal gray, amygdala, hippocampus, septum, and mammillary bodies (see Menard $\&$ Treit, 1999). It is, therefore, very unlikely that the mSuM is crucial for anxiety in any global sense but, during the FI task, it is probably the major substrate for the action of benzodiazepines on behavioral inhibition.

If specific control of theta frequency by the $\mathrm{mSuM}$ is related mainly to a behavioral inhibition function of the hippocampus, the $\mathrm{mSuM}$ is probably less involved in determining frequency during hippocampal-mediatedbehaviors for which behavioral inhibition is less critical. The Morris water maze is a hippocampal-sensitive task, that has a behavioral inhibition component (Gray \& McNaughton, 2000) but is generally regarded as a test for spatial memory ability. Pan and McNaughton (1997) found only small effects of $\mathrm{mSuM} / \mathrm{CDP}$ on water maze learning and theta frequency, despite the robust effects on both variables with other treatments (systemic CDP, long cooling). Clearly, theta frequency is critical for correct water maze performance, but the $\mathrm{mSuM}$ is not the only site that determines frequency during this task. There is other evidence for more than one nucleus' controlling the frequency of theta in awake rats. Lesions of the $\mathrm{mSuM}$ and surrounding areas do not alter the frequency of theta recorded during simple movement (Thinschmidt, Kinney, \& Kocsis, 1995), and the frequency of RPO-elicited theta depends only partially on the mSuM in freely moving animals (McNaughton et al., 1995). The involvement of the mSuM in any hippocampal-sensitivetask, therefore, may depend on the contribution the mSuM makes to the control of theta frequency in that task, relative to other sites. The site controlling theta frequency appears to depend on the nature of the task: The mSuM is extensively involved in FI theta, uninvolved in simple movement theta, and jointly involved with some other site or sites in the control of theta in the water maze. From our results, as well as from those of Pan and McNaughton (1997), it would appear that, regardless of which specific sites control theta frequency in a given situation, the degree to which any manipulation affects frequency will affect the degree of disruption of hippocampal-mediated behaviors. 


\section{REFERENCES}

Ammassari-Teule, M., Maho, C., \& Sara, S. J. (1991). Clonidine reverses spatial learning deficits and reinstates $\theta$ frequencies in rats with partial fornix section. Behavioural Brain Research, 45, 1-8.

Apostol, G., \& Creutzfeldt, O. D. (1974). Cross-correlation between the activity of septal units and hippocampal EEG during arousal. Brain Research, 67, 65-75.

Beatty, W. W., \& Schwartzbaum, J. S. (1968). Commonality and specificity of behavioral dysfunctions following septal and hippocampal lesions in rats. Journal of Comparative \& Physiological Psychology, 66, 60-68.

Beck, C. H. M., \& Fibiger, H. C. (1995). Conditioned fear-induced changes in behaviour and in expression of the immediate early gene c-fos with and without diazepam pretreatment. Journal of Neuroscience, 15, 709-720.

Bennett, T. L. (1975). The electrical activity of the hippocampus and the process of attention. In R. L. Isaacson \& K. H. Pribram (Eds.), The hippocampus: Vol. 2. Neurophysiologyand behavior (pp. 71-99). New York: Plenum.

Black, A. H., \& Young, G. A. (1972). Electrical activity of the hippocampus and cortex in dogs operantly trained to move and to hold still. Journal of Comparative \& Physiological Psychology, 79, 128-141.

BLAND, B. H. (1986). The physiology and pharmacology of hippocampal formation theta rhythms. Progress in Neurobiology, 26, 1-54.

Bland, B. H., \& Vanderwolf, C. H. (1972). Diencephalic and hippocampal mechanisms of motor activity in the rat: Effects of posterior hypothalamic stimulation on behaviour and hippocampal slow wave activity. Brain Research, 43, 67-88.

Borhegyi, Z, Magloczky, Z., Acsady, Z, \& Freund, T. F. (1998). The supramammillary nucleus innervates cholinergic and GABAergic neurons in the medial septum-diagonal band of Broca complex. Neuroscience, 82, 1053-1065.

BrazhNiK, E. S., \& Vinogradova, O. S. (1986). Control of the neuronal rhythmic bursts in the septal pacemaker of theta rhythm: Effects of anaesthetic and anti-cholinergic drugs. Brain Research, 380, 94-106.

Buzsaki, G., Leung, L. S., \& VANderwolf, C. H. (1983). Cellular bases of hippocampal EEG in behaving rats. Brain Research Reviews, 6, 139-171.

Destrade, C. (1982). Two types of diencephalically driven RSA (theta) as a means of studying memory formation in mice. Brain Research, 234, 486-493.

ElAZAR, A., \& ADEY, W. R. (1967). Electroencephalographic correlates of learning in subcortical and cortical structures. Electroencephalography \& Clinical Neurophysiology, 23, 306-319.

Ellen, P., \& Powell, E. W. (1962). Effects of septal lesions on behaviour generated by positive reinforcement. Experimental Neurology, $\mathbf{6}$, $1-11$.

Givens, B., \& Olton, D.S. (1994). Local modulation of basal forebrain: Effects on working and reference memory. Journal of Neuroscience, 14, 3578-3587.

Gogolak, G., Stumpf, C., Petsche, H., \& Sterc, J. (1968). The firing pattern of septal neurons and the form of the hippocampal theta wave. Brain Research, 7, 201-207.

Graeff, F. G., Quintero, S., \& Gray, J. A. (1980). Median raphe stimulation, hippocampal theta rhythm and threat-induced behavioral inhibition. Physiology \& Behavior, 25, 253-261.

Grastyan, E., Karmos, G., Vereczkey,L., \& Kellenyi, L. (1966). The hippocampal electrical correlates of the homeostatic regulation of motivation. Electroencephalography\& Clinical Neurophysiology, 21, 34-53.

Grastyan, E., Lissak, K., Madarasz, I., \& Donhoffer, H. (1959). Hippocampal electrical activity during the development of conditioned reflexes. Electroencephalography \& Clinical Neurophysiology, 11, 409-430.

GraY, J. A. (1972). Effects of septal driving of the hippocampal theta rhythm on resistance to extinction. Physiology \& Behavior, 8, 481-490.

GraY, J. A., \& BALL, G. G. (1970). Frequency specific relation between hippocampal theta rhythm, behaviour and amobarbital action. Science, 168, 1246-1248.
Gray, J. A., \& McNaughton, N. (1983). Comparison between the behavioural effects of septal and hippocampal lesions. Neuroscience \& Biobehavioral Reviews, 7, 119-188.

Gray, J. A., \& McNaughton, N. (2000). The neuropsychology of anxiety: An enquiry into the functions of the septo-hippocampalsystem ( $2 \mathrm{nd}$ ed.). Oxford: Oxford University Press.

Green, J. D., \& ARduini, A. A. (1954). Hippocampal electrical activity in arousal. Journal of Neurophysiology, 17, 533-557.

HADDAD, R. K., \& RABE, A. (1969). Modified temporal behavior in rats after large hippocampal lesions. Experimental Neurology, 23, 310317.

Hayakawa, T., ITo, H., \& Zyo, K. (1993). Neuroanatomical study of afferent projections to the supramammillary nucleus of the rat. Anatomy \& Embryology, 188, 139-148.

Kemp, R, \& KAADA, B. R. (1975). The relation of hippocampal theta activity to arousal, attentive behavior and somato-motor movements in unrestrained cats. Brain Research, 95, 323-342.

KIRK, I. J. (1998). Frequency modulation of hippocampal theta by the supramammillary nucleus and other hypothalamo-hippocampal interactions: Mechanisms and functional implications. Neuroscience \& Biobehavioral Reviews, 22, 291-302.

KIRK, I. J., \& McNaughton, N. (1991). Supramammillary cell firing and hippocampal rhythmical slow activity. NeuroReport, 2, 723-725.

KIRK, I. J., \& MCNAUghton, N. (1993). Mapping the differential effects of procaine on frequency and amplitude of reticularly elicited hippocampal rhythmical slow activity. Hippocampus, 3, 517-525.

Kramis, R. C., Vanderwolf, C. H., \& Bland, B. H. (1975). Two types of hippocampal rhythmical slow activity in both the rabbit and the rat: Relations to behavior and effects of atropine, diethyl ether, urethane and pentobarbital. Experimental Neurology, 49, 58-85.

LANDFIELD, P. W. (1977). Different effects of post-trial driving or blocking of the theta rhythm on avoidance learning in rats. Physiology \& Behavior, 18, 439-445.

Landfield, P. W., McGaugh, J. L., \& Tusa, R. J. (1972). Theta rhythm: A temporal correlate of memory storage processes in the rat. Science, $175,87-89$

Lee, M. G., Chrobak, J. J., Sik, A., Wiley, R. G., \& BuZsaki, G. (1994). Hippocampal theta activity following selective lesion of the septal cholinergic system. Neuroscience, 62, 1033-1047.

Manning, F. J., \& McDonough, J. H. (1974). Reinforcement omission, noncontingent reinforcement, and limbic lesions in rats. Behavioural Biology, 11, 327-338.

McNaughton, N., \& Coop, C. F. (1991). Neurochemically dissimilar anxiolytic drugs have common effects on hippocampal rhythmical slow activity. Neuropharmacology, 30, 855-863.

McNaughton, N., Logan, B., Panickar, K. S., Kirk, I. J., Pan, W. X., Brown, N. T., \& HeEnan, A. (1995). Contribution of synapses in the medial supramammillary nucleus to the frequency of hippocampal theta rhythm in freely moving rats. Hippocampus, 5, 534-545.

McNaughton, N., \& Morris, R. G. M. (1987). Chlordiazepoxide, an anxiolytic benzodiazepine, impairs place navigation in rats. Behavioural Brain Research, 24, 39-46.

McNaughton, N., Richardson, J., \& Gore, C. (1986). Reticular elicitation of hippocampal slow waves: Common effects of some anxiolytic drugs. Neuroscience, 19, 899-903.

McNaughton, N., \& Sedgwick, E. M. (1978). Reticular stimulation and hippocampal theta rhythm in rats: Effects of drugs. Neuroscience, 2 , 629-632.

Menard, J., \& Treit, D. (1999). Effects of centrally administered anxiolytic compounds in animal models of anxiety. Neuroscience \& Biobehavioral Reviews, 23, 591-613.

Miller, R. (1991). Cortico-hippocampal interplay and the representation of contexts in the brain. Berlin: Springer-Verlag.

Montoya, C. P., Heynen, A. J., Faris, P. D., \& Sainsbury, R. S. (1989). Modality specific type 2 theta production in the immobile rat. Behavioral Neuroscience, 103, 106-111.

Morris, R. G. M., \& Hagan, J. J. (1983). Hippocampal electrical activity and ballistic movement. In W. Seifert (Ed.), Neurobiologyof the hippocampus (pp. 321-331). London: Academic Press. 
Myers, R. D. (1966). Injection of solutions into cerebral tissue: Relation between volume and diffusion. Physiology \& Behavior, 1, 171-174.

Oddie, S. D., Stefanek, W., Kirk, I. J., \& Bland, B. H. (1996). Intraseptal procaine abolishes hypothalamic stimulation-induced wheelrunning and hippocampal theta activity in rats. Journal of Neuroscience, 16, 1948-1956.

OlsEN, R. W. (1982). Drug interactions at the GABA receptor-ionophore complex. Annual Review of Pharmacology \& Toxicology, 22, 245-277.

Pan, W. X., \& McNaughton, N. (1997). The medial supramammillary nucleus, spatial learning and the frequency of hippocampal theta activity. Brain Research, 764, 101-108.

Panickar, K. S., \& McNaughton, N. (1991). Effects of buspirone on fixed interval responding in rats. Journal of Psychopharmacology, $\mathbf{5}$, 410-417.

Paxinos, G., \& Watson, C. (1998). The rat brain in stereotaxic coordinates (2nd ed.). London: Academic Press.

Petsche, H., Stumpf, C., \& Gogolak, G. (1962). The significance of the rabbit's septum as a relay station between the midbrain and hippocampus: 1 . The control of hippocampus arousal activity by septum cells. Electroencephalography \& Clinical Neurophysiology, 19, 25-33.

Rawlins, J. N. P., Feldon, J., \& Gray, J. A. (1979). Septo-hippocampal connections and the hippocampal theta rhythm. Experimental Brain Research, 37, 49-63.

Rivas, J., Gaztelu, J. M., \& Garcia-Austt, A. E. (1996). Changes in hippocampal cell discharge patterns and theta rhythm spectral properties as a function of walking velocity in the guinea pig. Experimental Brain Research, 108, 113-118.

SAINSBURY, R. S. (1970). Hippocampal activity during natural behavior in the guinea pig. Physiology \& Behavior, 5, 317-324.

SAINSBURY, R. S. (1998). Hippocampal theta: A sensory-inhibition theory of function. Neuroscience \& Biobehavioral Reviews, 22, 237-241.

SAINSBURY, R. S., \& Bland, B. H. (1981). The effects of selective septal lesions on theta production in CA1 and the dentate gyrus of the hippocampus. Physiology \& Behavior, 26, 1097-1101.

Sandner, G., Oberling, P., Silveira, M. C., Di Scala, G., Rocha, B., BAgri, A, \& Depootere, R. (1993). What brain structures are active during emotions? Effects of stimulation elicited aversion on c-fos immunoreactivity and behaviour. Behavioural Brain Research, 58, 9-18.

Sano, K., Mayanagi, Y., Sekino, H., Ogashiwa, M., \& Ishijima, B. (1970). Results of stimulation and destruction of the posterior hypothalamus in man. Journal of Neurosurgery, 33, 689-707.

Shepard, P. D., Minailoff, G. A., \& German, D. C. (1988). Anatomical and electrophysiological characterization of presumed dopaminecontaining neurons within the supramammillary region of the rat. Brain Research Bulletin, 20, 307-314.

SLAWINSKA, U., \& KasicKI, S. (1998). The frequency of rat's hippocampal theta rhythm is related to the speed of locomotion. Brain Research, 796, 327-331.

Snape, M., Grigoryan, G., Sinden, J. D., \& Gray, J. A. (1996). Dependence of the proactive behavioral effects of theta-driving septal stimulation on stimulation frequency and behavioral experience: 2 . Continuously and partially reinforced running. Psychobiology, 24, 22-32.

Snedecor, G. W., \& Cochrane, W. G. (1967). Statisticalmethods. Ames: Iowa State University Press.

Stewart, M., \& Fox, S. E. (1989a). Detection of an atropine-resistant component of the hippocampal theta rhythm in urethane-anaesthetised rats. Brain Research, 500, 55-60.

StEWART, M., \& Fox, S. E. (1989b). Monkeys have hippocampal theta activity. Society for Neuroscience Abstracts, 15, 1250.

STUMPF, C. (1965). The fast component in the electrical activity of the rabbit's hippocampus. Electroencephalography \& Clinical Neurophysiology, 18, 477-486.

Thinschmidt, J. S., Kinney, G. G., \& Kocsis, B. (1995). The supramammillary nucleus: Is it necessary for the mediation of hippocampal theta rhythm? Neuroscience, 67, 301-312.

VANDERWOLF, C. H. (1969). Hippocampal electrical activity and voluntary movement in the rat. Electroencephalography \& Clinical Neurophysiology, 26, 407-418.

VERTES, R. P. (1980). Brain stem activation of the hippocampus: A role for the magnocellular reticular formation and the MLF. Electroencephalography \& Clinical Neurophysiology, 50, 48-58.

Vertes, R. P. (1986). Brainstem modulation of the hippocampus: Anatomy, physiology and significance. In R. L. Isaacson \& K. H. Pribram (Eds.), The hippocampus: Vol. 4 (pp. 41-75). New York: Plenum.

VERTES, R. P. (1988). Brainstem afferents to the basal forebrain in the rat. Neuroscience, 24, 907-935.

VERTES, R. P. (1992). PHA-L analysis of projections from the supramammillary nucleus in the rat. Journal of Comparative Neurology, $\mathbf{3 2 6}$ 595-622.

Vertes, R. P., \& Martin, G. F. (1988). Autoradiographic analysis of ascending projections from the pontine and mesencephalic reticular formation and the median raphe nucleus in the rat. Journal of Comparative Neurology, 275, 511-541.

VINOGRAdOVA, O. S (1995). Expression, control, and probable functional significance of the neuronal theta rhythm. Progress in Neurobiology, 45, 523-583.

Wetzel, W., Ott, T., \& Matthies, H. (1977). Post-training hippocampal rhythmic slow activity (theta) elicited by septal stimulation improves memory consolidation in rats. Behavioral \& Neural Biology, 21, 32-40.

Whishaw, I. Q., Bland, B. H., \& Vanderwolf, C. H. (1972). Hippocampal activity, behavior, self-stimulation, and heart rate during electrical stimulation of the lateral hypothalamus. Journal of Comparative \& Physiological Psychology, 79, 115-127.

Whishaw, I. Q., \& VANDERWOLF, C. H. (1973). Hippocampal EEG and behavior: Changes in amplitude and frequency of RSA (theta rhythm) associated with spontaneous and learned movement patterns in rats and cats. Behavioral Biology, 8, 461-484.

Williams, J. H., \& Gray, J. A. (1996). Dependence of the proactive behavioral effects of theta-driving septal stimulation on stimulation frequency and behavioral experience: 1 . Leverpress experiments. Psychobiology, 24, 9-21.

Winson, J. (1972). Interspecies differences in the occurrence of theta. Behavioral Biology, 7, 479-487.

WINSON, J. (1978). Loss of hippocampal theta rhythm results in spatial memory deficit in the rat. Science, 201, 160-163.

Woodnorth, M.-A., Kyd, R. J., Logan B. J., Long, M. A., \& McNaughton, N. (2002). Multiple hypothalamic sites control the frequency of hippocampal theta rhythm. Manuscript submitted for publication.

ZAR, J. H. (1974). Biostatistical analysis. Englewood Cliffs, NJ: PrenticeHall.

Zhu, X. O., \& McNaughton, N. (1994). The interaction of serotonin depletion with anxiolytics and anti-depressants on reticular-elicited hippocampal RSA. Neuropharmacology, 33, 1597-1605.

Zhu, X.O., \& McNaughton, N. (1995). Similar effects of buspirone and chlordiazepoxide on a fixed interval schedule with long-term, low-dose administration. Journal of Psychopharmacology, 9, 326-330.

(Manuscript received October 15, 2001; accepted for publication February 21, 2002.) 